\title{
Propiedades de Mezclas Extrudidas a Base de Polietileno de Baja Densidad y Polianilina
}

\author{
Carlos V. Cupul, Ricardo H. Cruz*, Alejandra Caballero y Luis M. Rangel \\ Centro de Investigación Científica de Yucatán, Unidad de Materiales, Calle 43, No. 130, \\ Colonia Chuburná de Hidalgo, 97200 Mérida, Yucatán-México (e-mail: rhcruze@cicy.mx)
}

*autor a quien debe ser dirigida la correspondencia

\begin{abstract}
Resumen
Se estudia el efecto de la adición de un complejo electro-conductor de polianilina (PANIPOL) sobre la conductividad eléctrica, la resistencia mecánica y la microestructura de materiales compuestos poliméricos. El análisis se realizó en extrudidos de polietileno de baja densidad-PANIPOL, con diferentes contenidos del relleno conductor, los cuales fueron obtenidos en forma de filamento mediante extrusión capilar. La conductividad volumétrica se calculó a partir de la resistencia eléctrica usando un electrómetro. La resistencia mecánica se evaluó con una máquina de pruebas universales y la microestructura se observó por microscopía electrónica de barrido. Los resultados indican que la conductividad aumenta, y la tenacidad a la ruptura disminuye con el contenido de PANIPOL. EI PANIPOL adquirió una microestructura en forma de estructuras alargadas en algunos extrudidos. Se concluye que es posible obtener materiales compuestos poliméricos con resistencia mecánica y conductividades adecuadas en el intervalo de los materiales antiestáticos utilizando contenidos bajos de PANIPOL.
\end{abstract}

\section{Properties of Extruded Blends Based on Low Density Polyethylene and Polyaniline}

\begin{abstract}
The effect of adding an electro-conducting polyaniline complex (PANIPOL) on the electrical conductivity, the mechanical resistance and the microstructure of polymer composites is studied. The analysis was performed on low density polyethylene-PANIPOL extruded blends, with different conducting filler contents. The filament-like composites were produced by capillary extrusion. The volume conductivity was calculated from the electrical resistance, which was measured with an electrometer. The mechanical resistance was evaluated with a universal testing machine, and the microstructure was observed by means of scanning electron microscopy. The results indicate that the conductivity increases, whereas the breaking tenacity decreases with the PANIPOL content. The PANIPOL microstructure was observed to be in the form of elongated structures in some extruded blends. It is concluded that it is possible to obtain polymer composites with adequate mechanical strength and conductivities within the range of those of antistatic materials using low PANIPOL contents.
\end{abstract}

Keywords: electro-conducting materials, polymers, polyaniline, mechanical resistance 


\section{INTRODUCCION}

Hace varios años los polímeros se consideraban típicamente aislantes. Sin embargo, Heeger, Shirakawa y McDiarmid demostraron que al dopar cierto tipo de polímeros que contienen enlaces dobles conjugados, se incrementaba su conductividad eléctrica hasta niveles similares a la de algunos metales, transformándose de esta manera en polímeros intrínsecamente electro-conductores (ICPs por sus siglas en inglés) (Chiang et al., 1977; Shirakawa et al., 1977). Esta propiedad abre la posibilidad de crear otros tipos diferentes de materiales para aplicaciones especializadas. Por ejemplo, es posible la obtención de materiales compuestos poliméricos semiconductores a partir mezclas de polímeros aislantes (ej., polipropileno, polietileno de baja densidad, etc.) con ICPs (Crossman, 1985; Peng et al., 2007) para la protección contra descargas electrostáticas (ESD por sus siglas en inglés) (Anand et al., 1998; Virtanen et al., 1997). Los materiales compuestos poliméricos a base de ICPs poseen también otras aplicaciones potenciales tales como en la fabricación de dispositivos para el almacenamiento de energía, aparatos electro-ópticos y en la soldadura de plásticos, entre otras (Cruz-Estrada y Flokes, 2000).

Algunas desventajas importantes de los ICPs son su pobre estabilidad ambiental, la dificultad de su procesamiento por métodos convencionales (Pomfret et al., 2000) y sus bajas propiedades mecánicas. Sin embargo, estas limitaciones pueden ser superadas preparando mezclas y compuestos de ICPs con otros polímeros para obtener un producto adecuado para determinadas aplicaciones. En este sentido, para el buen diseño de un material compuesto polimérico electroconductor se requiere que exista un balance apropiado entre la conductividad eléctrica, las propiedades mecánicas y las condiciones de procesamiento (Omastová et al., 1999). Por lo tanto, para una aplicación determinada, siempre se está en la búsqueda de técnicas, métodos y condiciones de procesamiento adecuados que mejoren la conductividad y el control de ésta, así como también las propiedades mecánicas del producto final.

De entre toda la amplia gama de aplicaciones posibles de los materiales compuestos poliméricos electro-conductores, Ilaman especialmente la atención aquellos materiales capaces de ofrecer protección contra las descargas electrostáticas, las cuales pueden llegar a representar un peligro serio, además de que suponen ser una de las principales causas de pérdidas económicas a nivel mundial. Hoy en día, para resolver este problema se ofrecen nuevos materiales en cada área, y productos originales se están introduciendo constantemente al mercado. Entre éstos, son interesantes los materiales preparados a base de filamentos electro-conductivos embebidos en polímeros aislantes, los cuales se usan en la fabricación de textiles y alfombras con propiedades antiestáticas. La gran mayoría de los métodos empleados en la actualidad para la preparación de filamentos antiestáticos involucra la co-extrusión y/o co-fibrilado ("co-spinning" en inglés) de dos materiales para la formación de filamentos conocidos como los de tipo funda-núcleo ("sheath-core" en inglés), en los que el material que actúa como funda es un polímero aislante, mientras que el otro, es un material conductor (generalmente una mezcla polimérica a base de negro de humo) que actúa como un núcleo conductor alargado y orientado a todo lo largo del filamento (Blackmon et al., 1994; Boe, 1976; De Howitt, 1986; Green, 2000; Hull, 1974; Rasnick y Belcher, 2001; Rodini, 1991). Estos procesos pueden llegar a ser costosos y un tanto complejos, debido a la cantidad de equipos y accesorios que se requieren, y en especial, al acoplamiento sofisticado que existe entre ellos. Una técnica más simple y promisoria sería generar las fibras electro-conductivas in-situ, es decir, durante el mismo proceso de formado del filamento antiestático, para lo cual se emplearía un relleno conductor a base de un ICP como fase dispersa en el material compuesto. Esta estrategia involucraría la formación, mediante un solo proceso y equipo, de fibras electro-conductivas a base de un ICP embebidas en el seno de una matriz polimérica aislante. Los procesos de deformación in-situ para la generación de fibras en sistemas poliméricos aislantes han sido intensivamente estudiados, y se ha demostrado que bajo ciertas condiciones de procesamiento y propiedades de los polímeros de la mezcla, la acción de la matriz sobre la fase dispersa puede ser tal que esta última se deforme de tal manera que se obtengan estructuras alargadas (ej., elipsoides, fibras, etc.) (Boyaud et al., 2001; Ehtaiatkar et al., 1989; Folkes y Reip, 1986; Tjong y Jiang, 2004; Tsebrenko et al., 1976).

Con respecto al relleno conductor, se sabe que la polianilina dopada (PANI) ha sido uno de los ICPs más empleados debido a su excelente estabilidad química y a sus niveles relativamente altos de 
conductividad. Al respecto, existen en el mercado complejos conductores a base de PANI, los cuales pueden procesarse fácilmente por métodos térmicos como la extrusión debido a que contienen ciertos aditivos que disminuyen la viscosidad de sus mezclas con otros polímeros termoplásticos durante su procesamiento, lo que es una ventaja en comparación con otros tipos de rellenos conductivos (ej., negro de humo, partículas metálicas, etc.) (Kärnä et al., 1994a, 1994b; Virtanen et al., 1997). Además, estos complejos son solubles en ciertos solventes de uso común, y pueden también ser utilizados en la preparación de mezclas poliméricas conductivas con límites muy bajos de percolación para obtener materiales semiconductores con niveles de conductividad en el intervalo óptimo reportado para la protección contra ESD (Perento, 2004).

En trabajos previos se estableció la viabilidad de usar un proceso de deformación in-situ para generar estructuras alargadas de un complejo conductor a base de PANI (PANIPOL) que estuvieran embebidas en el seno de una matriz de poli(estireno-butadieno-estireno) (Cruz-Estrada y Folkes, 2000; Cruz-Estrada y Cupul-Manzano, 2005). El presente trabajo trata sobre el mismo tema. Sin embargo, se reporta ahora sobre la experimentación llevada a cabo con polietileno de baja densidad (LDPE por sus siglas en inglés) como matriz polimérica aislante para la preparación de materiales poliméricos semiconductores en forma de filamento con aplicaciones potenciales en la protección contra ESD. De tal manera, en este trabajo se reporta la obtención de compuestos electroconductores a base de LDPE y PANI mediante extrusión capilar, así como su caracterización. También se explora la posibilidad de que el relleno conductor se deforme en estructuras alargadas (idealmente en microfibras extendidas) en el interior del material compuesto sin que se requieran etapas posteriores de procesamiento.

\section{METODOLOGÍA}

\section{Materiales}

Se elaboraron mezclas binarias utilizando LDPE como matriz (Lupolen $1810 \mathrm{E}$ de Basf, temperatura de fusión $=108^{\circ} \mathrm{C}$, densidad $=919 \mathrm{~kg} / \mathrm{m}^{3}$ ) y un complejo conductor de polianilina (PANIPOL) como relleno conductor (clave CXL300X35 de Panipol Ltd, temperatura máxima de procesamiento = $230^{\circ} \mathrm{C}$, intervalo de temperaturas de procesamiento recomendadas $=100-225^{\circ} \mathrm{C}$, conductividad volumétrica típica $=1 \times 10^{-4} \mathrm{~S} / \mathrm{cm}$, densidad $\left.=1100 \mathrm{~kg} / \mathrm{m}^{3}\right)$. Este material tiene una composición aproximada de $25 \%$ en peso de polianilina electro-conductiva y $75 \%$ en peso de dodecil-bencen sulfonato de zinc (Cruz-Estrada, 2004). EI PANIPOL es higroscópico, por lo que se secó durante 48 hrs a $60^{\circ} \mathrm{C}$ antes de ser utilizado. El polímero que se empleó como matriz no recibió ningún tratamiento previo al mezclado.

\section{Procesamiento}

Se prepararon mezclas con contenidos de $1,5,10,15,20,35$ y $50 \%$ en peso de PANIPOL en la matriz aislante de LDPE. Para formar las mezclas en filamentos, éstas se procesaron en un extrusor mono-husillo Brabender de laboratorio (Modelo No. 8 319) con dos zonas de calentamiento (relación longitud/diámetro $=20$, diámetro $=19 \mathrm{~mm}$ ), empleando un husillo con una razón de compresión de $2: 1$ y paso cuadrado. En el extremo de salida del extrusor se acopló un dado capilar (relación longitud/diámetro $=7.5$ ) de $2 \mathrm{~mm}$ de diámetro interno. El sistema de procesamiento incluyó un baño de agua corriente a temperatura ambiente situado a la salida del dado capilar y un sistema jalador de rodillos con velocidad variable. Todas las mezclas se procesaron a una velocidad de rotación del husillo de $5 \mathrm{rpm}$. El perfil de temperaturas empleado para el procesamiento fue de $120^{\circ} \mathrm{C}$ en el barril del extrusor y $110^{\circ} \mathrm{C}$ en la zona del dado. Para fines comparativos, también se obtuvieron extrudidos tanto de LDPE, como de PANIPOL puros siguiendo el método de procesamiento ya descrito. El diámetro aproximado de los extrudidos obtenidos estuvo entre 0.9 y $1.6 \mathrm{~mm}$.

\section{Evaluación de la conductividad volumétrica}

La conductividad volumétrica de los filamentos se calculó a partir de su resistencia eléctrica, la cual se midió empleando un electrómetro Keithley, Modelo 2400C. Para obtener la resistencia eléctrica se cortaron 10 muestras de $5 \mathrm{~cm}$ de longitud de los extrudidos obtenidos de cada una de las diferentes 
mezclas procesadas. Las muestras se cortaron en forma aleatoria de las secciones continuas obtenidas, excluyendo los extremos. Se midió el diámetro promedio de cada muestra con la ayuda de un vernier digital para emplearlo en los cálculos. Aproximadamente $5 \mathrm{~mm}$ de los extremos de cada muestra se recubrieron con pintura de plata para disminuir la resistencia de contacto con los electrodos. Las muestras a probar se acondicionaron durante $48 \mathrm{hrs}$ a $50 \%+/-10 \%$ de humedad relativa y $21^{\circ} \mathrm{C}+/-2^{\circ} \mathrm{C}$, basándose en la norma estándar ASTM D-618 (2000). Las pruebas eléctricas se realizaron bajo las mismas condiciones.

La resistencia de cada muestra se calculó utilizando la ley de Ohm (Ecuación 1), por lo que se aplicaron diferentes niveles de voltaje en el intervalo de 1 a $210 \mathrm{~V}$ para verificar que éste variara en forma directamente proporcional con la intensidad de corriente a través de la muestra, y de esta manera, confirmar que se cumpliera la ley de Ohm. Así, la resistencia para cada nivel de voltaje aplicado se determinó mediante la siguiente ecuación:

$V=I R$

Donde $\mathrm{V}=$ voltaje aplicado en voltios $(\mathrm{V}), \mathrm{I}=$ intensidad de corriente a través de la muestra en amperios $(A)$ y $R=$ resistencia en ohmios $(\Omega)$. Posteriormente, se obtuvo una resistencia promedio para cada contenido de PANIPOL en el intervalo de voltajes en el cual se cumplía la ley de Ohm.

La conductividad volumétrica se calculó empleando la ecuación 2 (Crossman, 1985):

$\sigma=\frac{l}{R A}=\frac{4 l}{\pi d^{2} R}$

Donde $\sigma=$ conductividad volumétrica en siemens por $\mathrm{cm}(\mathrm{S} / \mathrm{cm}), \mathrm{R}=$ resistencia en ohmios, I = longitud de la muestra en $\mathrm{cm}, \mathrm{A}=$ área de la sección transversal en $\mathrm{cm}^{2}$, y $\mathrm{d}=$ diámetro de la muestra en $\mathrm{cm}$. Finalmente, se graficaron las conductividades volumétricas promedio de los extrudidos con respecto al contenido de PANIPOL en cada una de las diferentes mezclas estudiadas.

\section{Análisis de la microestructura}

La morfología adquirida por el relleno conductor en el seno de la matriz polimérica aislante de los extrudidos se observó de manera indirecta. Para esto, se llevó a cabo la extracción selectiva de una de las fases del material compuesto extrudido. De esta manera, sería posible observar por microscopía la forma de las marcas dejadas (o "huellas") sobre la superficie de las muestras analizadas al extraer uno de los componentes, y así relacionarlas con la morfología original del relleno conductor. El solvente elegido para la extracción fue el xileno. Para esto, se cortaron muestras al azar de aproximadamente $0.5 \mathrm{~cm}$ de longitud de los extrudidos. Todos los extrudidos se cortaron longitudinalmente con un escalpelo en dirección perpendicular a la de la extrusión de las mezclas, tratando en lo posible de que las áreas superficiales fueran similares en todas las muestras. Posteriormente, las muestras se depositaron en viales individuales, a los cuales se les agregó la cantidad de solvente apropiado (aproximadamente $4 \mathrm{ml}$ por vial) para asegurar la inmersión completa de éstas. La extracción se realizó durante 7 días sin agitación a temperatura ambiente. Seguidamente, se retiró el solvente, se lavaron las muestras con agua destilada y se dejaron secar en una estufa a $70^{\circ} \mathrm{C}$ durante $48 \mathrm{hrs}$. Finalmente, las muestras se analizaron por microscopía electrónica de barrido (SEM por sus siglas en inglés) utilizando un equipo marca JEOL 6360 LV. No se aplicó recubrimiento conductivo a las muestras para el análisis.

Debe mencionarse que tanto el complejo conductor de PANI, como el LDPE son disueltos a temperatura ambiente por solventes aromáticos tales como el xileno (el PANIPOL en mayor grado) (Davies et al., 1995; Hsu y Ho, 1997; Luzny et al., 1998; Vikki et al., 1996). Sin embargo, con el 
propósito de evaluar cual de estos dos materiales sería más susceptible a ser disuelto, se llevó a cabo un experimento en el cual tanto extrudidos de PANIPOL, como extrudidos de LDPE puros con áreas superficiales similares, fueron respectivamente sometidos al mismo proceso de extracción en xileno aplicado al resto de los extrudidos. Este análisis se llevó a cabo por triplicado para cada tipo de material. Tanto los extrudidos de LDPE como los de PANIPOL, sometidos al mismo proceso de extracción, fueron también analizados por SEM para fines comparativos.

\section{Evaluación de la resistencia mecánica}

De los extrudidos obtenidos se cortaron 5 muestras al azar con una longitud aproximada de $21 \mathrm{~cm}$, determinándoseles el diámetro promedio. La densidad lineal requerida por los cálculos se obtuvo con base en la norma ASTM D-1577 (2001) (opción A). Las muestras se acondicionaron en atmósfera estándar ( $50 \pm 5 \%$ de humedad relativa y $23 \pm 2^{\circ} \mathrm{C}$ de temperatura) durante al menos 40 hrs según la norma ASTM D-618 (2000). La resistencia mecánica fue evaluada a tracción en una máquina de pruebas universales Shimadzu AGI, utilizando mordazas neumáticas, a una velocidad de cabezal de $475 \mathrm{~mm} / \mathrm{min}$ (muy próxima a la máxima del equipo) y con una apertura entre mordazas de $10 \mathrm{~mm}$ (debido al estiramiento esperado). Se evaluó la tenacidad a la ruptura ("breaking tenacity" en inglés), entendiéndose ésta como la fuerza máxima soportada por la muestra llevada a ruptura con respecto a su densidad lineal.

\section{RESULTADOS Y DISCUSION}

\section{Evaluación de la conductividad volumétrica}

En general, el voltaje aplicado a los extrudidos varió en forma directamente proporcional con respecto a la intensidad de corriente medida a través de las muestras, principalmente en el intervalo de 1 a 170 voltios. La resistencia eléctrica de cada muestra fue calculada empleando niveles de voltaje comprendidos en el intervalo antes señalado y empleando la ecuación 1.

En la Fig. 1 se muestra la variación de la conductividad volumétrica de los filamentos LDPEPANIPOL con respecto al porcentaje en peso del relleno conductor. Los valores extremos de la gráfica, es decir, las conductividades de las materias primas puras, fueron tomados de la literatura (Brandrup et al., 1999; Cruz-Estrada, 2004).

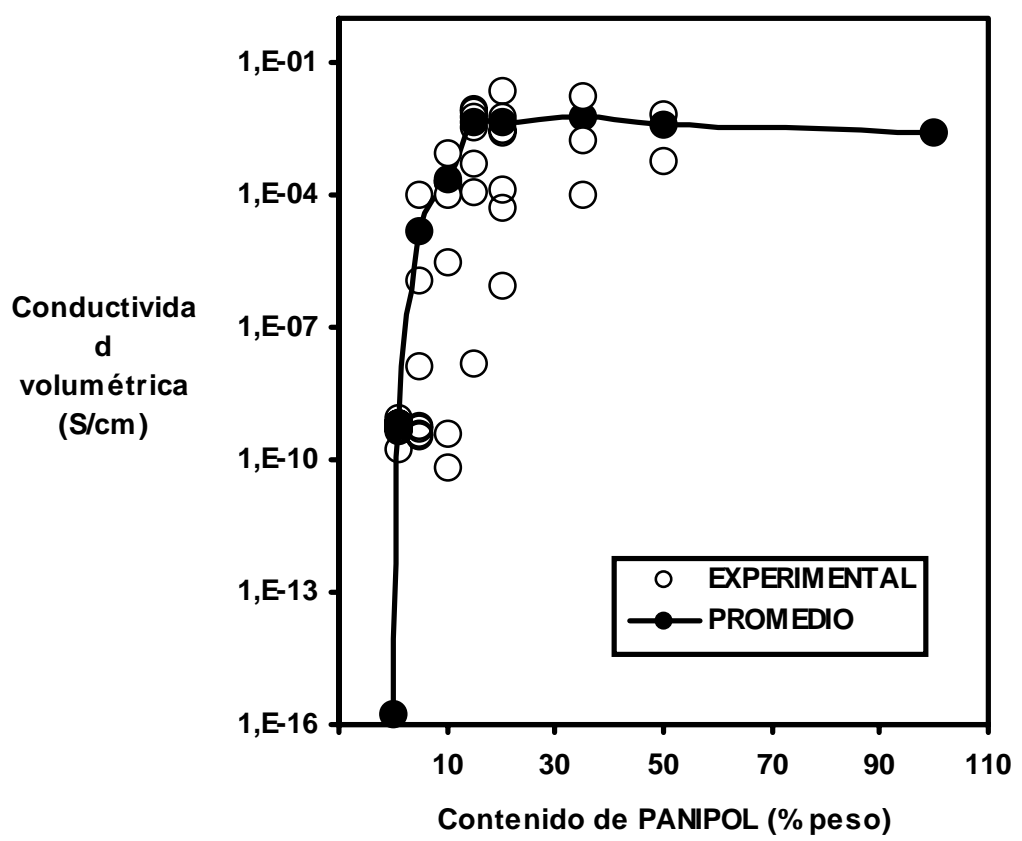

Fig. 1: Variación de la conductividad volumétrica con respecto al contenido de PANIPOL en extrudidos PANIPOL-LDPE. 
En la Figura 1 se observa que la variación de la conductividad de los extrudidos con respecto al contenido del relleno conductor presenta el comportamiento general característico de un sistema de percolación para materiales compuestos poliméricos a base de polianilina (Anand et al., 1998). Así, se nota un incremento súbito de por lo menos 6 órdenes de magnitud de la conductividad del extrudido con $1 \%$ en peso de PANIPOL con respecto a la del LDPE. Se considera que para contenidos muy bajos del relleno conductor, la separación entre las partículas de PANIPOL es relativamente grande por lo que no se obtienen niveles altos de conductividad. Posteriormente, es posible que al incrementar gradualmente el contenido de las partículas conductoras en el material, ocurra la formación de los primeros "caminos electro-conductores", debido al contacto de éstas entre si, ó posiblemente debido a la disminución de la separación entre ellas. Este mecanismo es el que se cree que ocurre en el intervalo de percolación. En la Figura 1 también se observa que la variación en los niveles de conductividad para los extrudidos con cantidades de PANIPOL mayores al $15 \%$ es prácticamente despreciable, con tendencia a la estabilización. Por lo tanto, es posible afirmar que la conductividad máxima para este sistema polimérico en particular se alcanza con el $15 \%$ de relleno conductor. Con esta cantidad, es posible que ocurra coalescencia de las partículas del relleno electro-conductor, lo cual posiblemente sea la causa por la que no se observa una variación significativa en la conductividad del material compuesto con contenidos mayores de PANIPOL. En la Figura anterior se nota también que la dispersión de los datos experimentales es alta, probablemente debido a que durante la elaboración de los extrudidos difícilmente se logró alcanzar un flujo estable de material que garantizara la obtención de filamentos completamente homogéneos. Al respecto, en la mayoría de los casos se observó que durante el procesamiento de las mezclas no se lograron obtener extrudidos completamente continuos del lote completo de la formulación, sino que se obtuvieron tramos de diferentes longitudes. Este hecho indica que el proceso de obtención debe ser optimizado.

En la Figura anterior se observa también que las conductividades calculadas para todos los extrudidos que contienen PANIPOL, generalmente quedan comprendidas en el intervalo de valores de conductividad de los semiconductores (Crossman, 1985; Peng et al., 2007; Perento, 2004), por lo que materiales de este tipo podrían emplearse como una alternativa en aplicaciones en las que se requieran estos niveles de conductividad y la flexibilidad mecánica característica de los polímeros termoplásticos. Por ejemplo, los extrudidos con contenidos aproximados de 1 a $5 \%$ en peso de PANIPOL poseen niveles de conductividad que están en el intervalo típico reportado de conductividades de los materiales empleados para la protección contra descargas electrostáticas (Perento, 2004).

\section{Análisis de la microestructura}

El análisis practicado a las secciones longitudinales de los extrudidos tratados con xileno reveló la presencia de morfologías alargadas (cavidades) paralelas a la dirección de la extrusión. Como ya se ha mencionado, tanto el PANIPOL como el LDPE son disueltos en cierto grado por el xileno. Sin embargo, los resultados del proceso de extracción en xileno al cual fueron respectivamente sometidos los extrudidos de PANIPOL y LDPE puros, indicaron que la pérdida promedio de peso en las muestras de PANIPOL fue $26.88 \%$. En contraste, la perdida promedio en las muestras de LDPE fue solamente $0.58 \%$ en peso. Si se asume que las áreas superficiales de las muestras de PANIPOL y LDPE fueron las mismas, estos resultados indican que la cantidad de PANIPOL perdida debido a la acción del solvente fue cerca de 46 veces mayor que la cantidad perdida de LDPE. Esto sugiere que el PANIPOL es más susceptible a ser disuelto por el xileno que el LDPE. Por lo tanto, el complejo conductor de PANI sería mayormente removido de la superficie de las secciones de los extrudidos PANIPOL-LDPE, dejando a la matriz de LDPE expuesta. Esto significa que lo que se observaría en la superficie de las muestras serían principalmente las "huellas" alargadas (es decir, cavidades alargadas) del complejo conductor de polianilina, el cual fue retirado por el solvente de la matriz de LDPE.

Por conveniencia, solamente se presentan micrografías correspondientes a los extrudidos con 15 y $20 \%$ en peso de PANIPOL (Figura 2). En la Figura 2a se muestra la sección longitudinal de un extrudido con $15 \%$ del relleno conductor, el cual fue tratado con xileno. Se observa la formación de "surcos" de corta longitud, razonablemente bien orientados en la dirección de la extrusión, los cuales 
tienden a unirse para formar "cavidades" alargadas de mayor longitud, algunas de las cuales se encuentran en el interior del óvalo en color blanco señalado en la Figura.
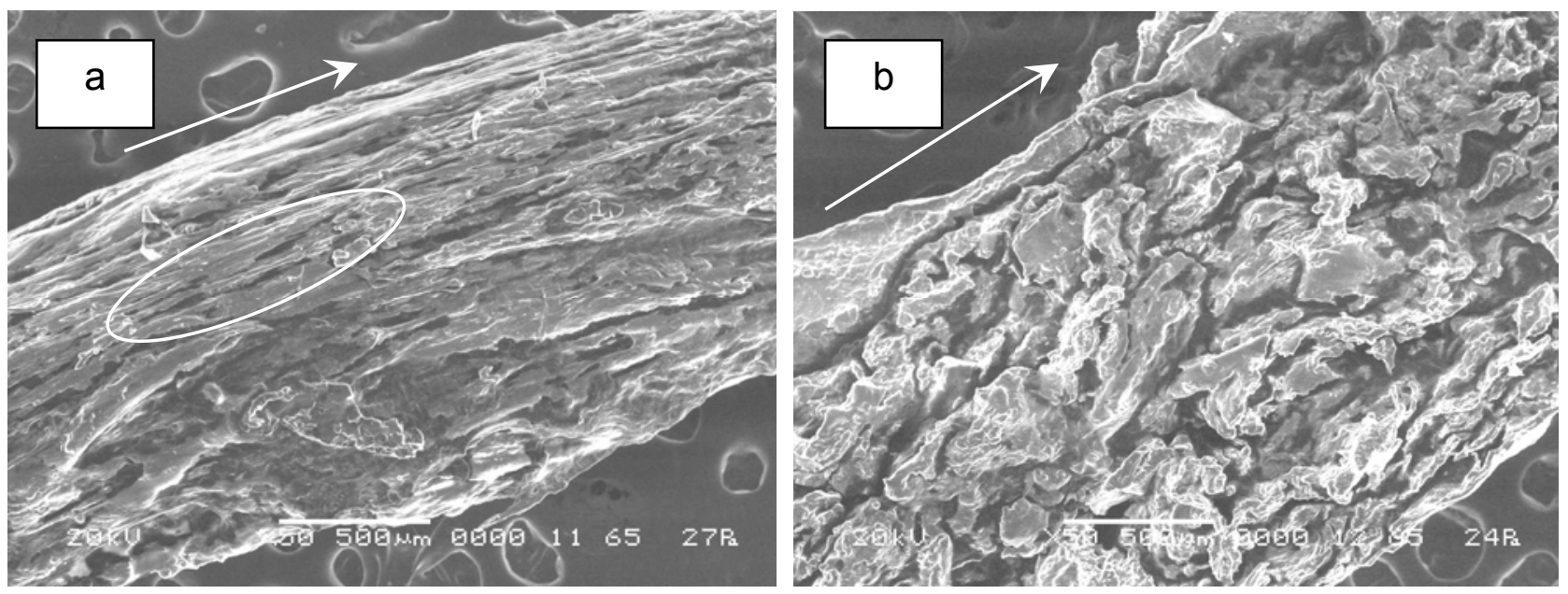

Fig. 2:. Micrografías SEM de la sección longitudinal de extrudidos PANIPOL-LDPE con 15\% (a) y $20 \%$ (b) en peso de PANIPOL tratados con xileno. Las flechas indican la dirección de la extrusión, y el óvalo en color blanco en Fig. 2a encierra algunas estructuras alargadas típicas.

En la Figura 2a también se aprecian agujeros relativamente grandes, en donde presumiblemente el relleno conductor no se distribuyó adecuadamente, permaneciendo en forma de grumos de diferentes extensiones, debido probablemente a la coalescencia de las estructuras del relleno conductor.

Es muy probable que el material extraído en el extrudido referido en el párrafo anterior sea PANIPOL en su mayor parte, ya que como se ha demostrado, el PANIPOL es más susceptible a ser disuelto por el xileno en comparación con el LDPE. Por lo tanto, resulta evidente que la morfología observada en el extrudido PANIPOL-LDPE fue resultado de la presencia del complejo electro-conductivo, el cual originalmente tuvo la forma de algunas estructuras alargadas embebidas en el seno del material compuesto.

Por otro lado, en la Figura $2 \mathrm{~b}$ se observa la sección longitudinal de un extrudido con $20 \%$ del relleno conductor, tratado con xileno. Se puede notar que la superficie expuesta presenta una elevada porosidad debido a la extracción. Los surcos y agujeros formados tienden a orientarse en menor medida en la dirección de la extrusión ya que, probablemente, la mayor cantidad de PANIPOL en la mezcla extrudida causó coalescencia, de manera que se formaran grumos, los cuales se unieran para finalmente formar una fase co-continua con la matriz. Se cree que lo anterior ocurre en mezclas extrudidas con mayor cantidad de relleno conductor.

En contraste, al analizar la superficie de la sección longitudinal de un extrudido de LDPE tratado con xileno (Figura 3), se observó que presentó morfologías diferentes a las observadas en la micrografía de la Figura 2a. Es decir, no se observaron las cavidades alargadas con el mismo tipo de morfología de aquellas observadas en el extrudido con $15 \%$ de PANIPOL. Lo anterior demuestra que es viable la formación in-situ de estructuras alargadas de un complejo conductor de polianilina orientadas en la dirección del proceso de extrusión, especialmente si se comparan las morfologías de las secciones longitudinales de los extrudidos con y sin PANIPOL.

Es posible entonces que la formación de estructuras fibrilares, en los extrudidos PANIPOL-LDPE estudiados, ocurra para contenidos de relleno conductor menores al $15 \%$, ocasionando que aumente la conductividad rápidamente, para posteriormente estabilizarse debido a la formación de grumos de PANIPOL. 


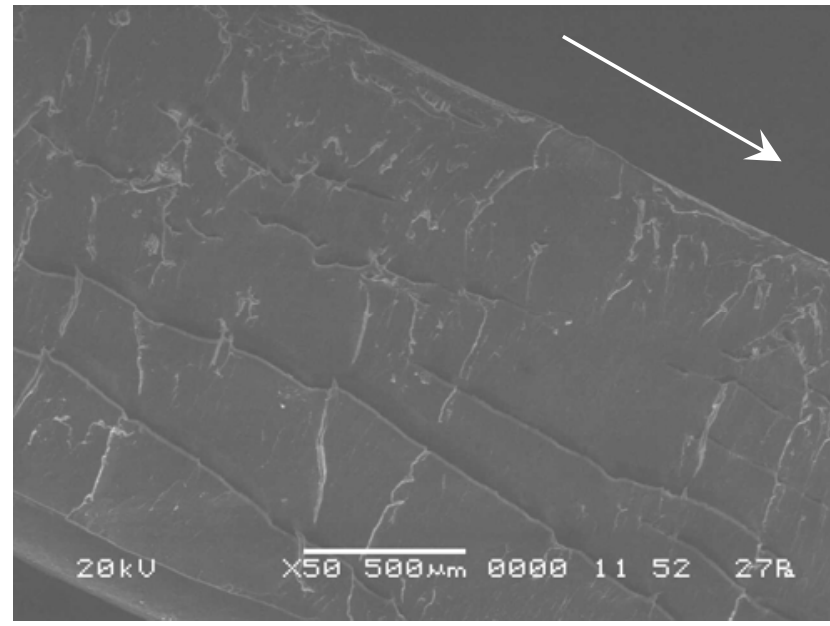

Fig. 3: Micrografía SEM de la sección longitudinal de un extrudido de LDPE tratado con xileno. La flecha indica la dirección de la extrusión.

\section{Evaluación de la resistencia mecánica}

La Figura 4 muestra la variación del promedio de la tenacidad a la ruptura con el contenido en peso de PANIPOL. En la Figura se observa que en general la tenacidad a la ruptura disminuye conforme se incrementa la cantidad del relleno conductor, lo cual es de esperarse, ya que sus propiedades mecánicas son muy pobres en comparación con las del LDPE. Se aprecia también que las tenacidades de los extrudidos de LDPE y de los que contienen 1 y $5 \%$ de PANIPOL son relativamente similares, lo cual podría deberse a que el contenido de PANIPOL en ellos no es tan significativo como para afectar sus tenacidades, ocasionando que prácticamente se comporten mecánicamente como el LDPE. Para contenidos mayores de PANIPOL se observa un decaimiento abrupto de la tenacidad hasta ser prácticamente nula (cero) para los extrudidos con contenidos de PANIPOL de 15 a $50 \%$, y PANIPOL puro. La disminución de la tenacidad a la ruptura de los extrudidos los hace menos resistentes a las fuerzas de tracción, e indica que la carga mecánica máxima que éstos pueden soportar antes de que ocurra la ruptura es cada vez menor conforme se incrementa el contenido de PANIPOL en ellos. Sería de esperarse que los extrudidos tiendan también a ser deformados con mayor facilidad a niveles más bajos de tracción al incrementar el contenido del relleno electro-conductor en ellos.

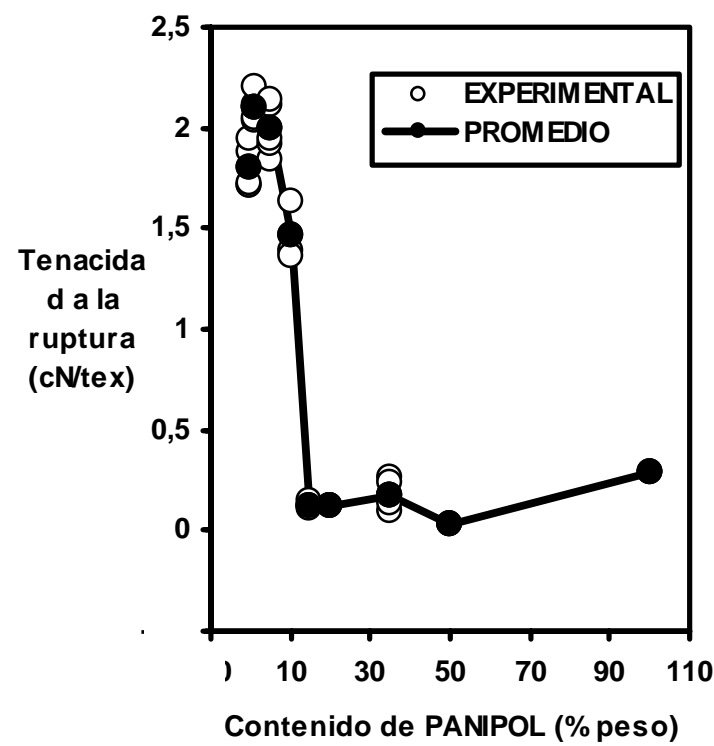

Fig. 4: Variación de la tenacidad a la ruptura con el contenido de PANIPOL en extrudidos PANIPOL-LDPE. 
De estos resultados, es interesante notar que la tenacidad a la ruptura de los extrudidos cuyas conductividades estuvieron en el intervalo óptimo reportado para protección contra descargas electrostáticas (extrudidos con contenidos de 1 a $5 \%$ en peso de PANIPOL) fue prácticamente la misma que la del extrudido de LDPE puro, lo cual podría ser una ventaja para una posible aplicación en la fabricación de productos antiestáticos, como por ejemplo alfombras y textiles, ya que tendrían la resistencia mecánica adecuada, al menos en lo que a tracción se refiere. Sería interesante sin embargo, estudiar cómo se comportarían mecánicamente estos materiales al someterlos a algún tipo de cargas compresivas. Esto será tema de investigación posterior, junto con otros aspectos de interés.

\section{CONCLUSIONES}

La variación de la conductividad volumétrica de extrudidos PANIPOL-LDPE con respecto al contenido del relleno electro-conductor presentó un comportamiento que en términos generales es característico de un sistema de percolación. Los materiales compuestos poliméricos electroconductivos obtenidos presentaron valores de conductividad comprendidos entre los de los semiconductores, un hecho que puede explotarse a futuro para el diseño de materiales para este tipo de aplicaciones. Al respecto, los extrudidos con contenidos aproximados de 1 a $5 \%$ en peso de PANIPOL tuvieron niveles de conductividad que están en el intervalo típico reportado de conductividades de los materiales empleados para la protección contra descargas electrostáticas.

Los resultados de los experimentos de la extracción selectiva de la fase electro-conductora con xileno, junto con el análisis microscópico de los extrudidos, sugirieron que el relleno conductor tendió a formar pequeñas estructuras alargadas en los materiales compuestos con contenidos bajos de éste, las cuales se orientaron principalmente en la dirección de la extrusión. Conforme aumentó el contenido de PANIPOL, estas estructuras tendieron a unirse para formar otras más largas, o a unirse lateralmente para formar grumos (coalescencia). En general, estos resultados demuestran que es viable la formación de estructuras alargadas de un complejo conductor de polianilina en una matriz de LDPE, orientadas a todo lo largo de extrudidos en forma de filamento mediante un simple proceso de deformación in-situ.

La tenacidad a la ruptura de los extrudidos PANIPOL-LDPE disminuyó en general con el incremento del relleno conductor. Específicamente, la tenacidad de los extrudidos con contenidos bajos de PANIPOL (1-5 \%) fue relativamente similar a la del extrudido de LDPE, lo cual pudo deberse a que el contenido del relleno conductor en ellos no fue tan significativo lo cual ocasionó que prácticamente se comportaran mecánicamente como la matriz polimérica aislante. Es interesante el hecho de que la tenacidad de los extrudidos cuyas conductividades estuvieron en el intervalo óptimo reportado para protección contra descargas electrostáticas fue prácticamente la misma que la del extrudido de LDPE puro, lo cual podría ser una ventaja para una posible aplicación en la fabricación de alfombras y textiles antiestáticos.

Los autores están conscientes de que se debe estudiar con más detalle el efecto que ejerce el método de procesamiento de las mezclas sobre la conductividad y, muy en particular, el efecto de las operaciones de estirado de los filamentos a la salida del dado de extrusión. De igual manera, se debe controlar mejor la velocidad y fuerza del jalado durante el proceso de elaboración de los extrudidos, ya que se piensa que esto influye de manera importante en las propiedades mecánicas.

\section{AGRADECIMIENTOS}

Al CONACyT el apoyo financiero para llevar a cabo este trabajo a través del proyecto SEP-2004C01-46395 y a la Ing. Química Silvia Beatriz Andrade Canto por la toma de las fotografías en el microscopio electrónico de barrido.

\section{REFERENCIAS}

Anand, J., S. Palaniappan y D.N. Sathyanarayana; Conducting polyaniline blends and composites, Prog. Polym. Sci.: 23, 993-1018 (1998). 
ASTM D-618; Standard practice for conditioning plastics for testing, ASTM International, 36-39, Philadelphia, Pa, USA (2000).

ASTM D-1577; Standard test method for linear density of textile fibers, ASTM International, 408-417, Philadelphia, Pa, USA (2001).

Blackmon, L.E., J.D. Forster y W.J. Nunning; Process for Forming a Yarn Having at least one Electrically Conductive Filament by Simultaneously Cospinning Conductive and Non-Conductive Filaments, US 5.277.855, Clase U.S. 264/103, 11 de enero (1994).

Boe, N.W.; Man-Made Textile Antistatic Strand, US 3.969.559, Clase U.S. 428/87, 13 de julio (1976).

Boyaud, M.F. y otros cuatro autores; Transient elongational properties of an in situ generated polymer/polymer composite, Polym. Eng. Sci.: 41 (4), 684-695 (2001).

Brandrup J, E.H. Immergut y E.A. Grulke; Polymer Handbook, Fourth edition, Volume 1, p v/17, John Wiley \& sons, Inc., New Jersey, U. S. A. (1999).

Chiang, C.K. y otros siete autores; Electrical conductivity in doped polyacetylene, Phys. Rev. Lett.: 39 (17), 1098-1101 (1977).

Crossman, R.A.; Conductive composites past, present, and future, Polym. Eng. Sci.: 25 (8), 507-513 (1985).

Cruz-Estrada, R.H.; On the characterization of an electrically conductive polyaniline complex, J. Mater. Sci.: 39, 511-518 (2004).

Cruz-Estrada, R.H. y C.V. Cupul-Manzano; Structure formation in polyaniline-based polymer blends, J. Mater. Sci.: 40 (24), 6571-6579 (2005).

Cruz-Estrada, R.H. y M.J. Folkes; In Situ production of electrically conductive fibres in polyanilineSBS blends, J. Mater. Sci.: 35, 5065-5069 (2000).

Davies, S.J., T.G. Ryan, C.J. Wilde y G. Beyer; Processable forms of conductive polyaniline, Synth. Met.: 69, 209-210 (1995).

De Howitt, J.R.; Process for Combining and Codrawing Antistatic Filaments with Undrawn Nylon Filaments, US 4.612.150, Clase U.S. 264/103, 16 de septiembre (1986).

Ehtaiatkar, F., M.J. Folkes, y S.C. Steadman; In situ production of polyethylene fibres from polymer blends, J. Mater. Sci.: 24, 2808-2814 (1989).

Folkes, M.J. y P.W. Reip; SBS block copolymer-polystyrene blends: 1. Morphology and swelling properties, Polymer: 27, 377-383 (1986).

Green, J.R.; Yarns Suitable for Durable Light Shade Cotton/Nylon Clothing Fabrics containing Carbon Doped Antistatic Fibers, US 6.057.032, Clase U.S. 428/359, 2 de mayo (2000).

Hsu, W.P. y K.S. Ho; Solvent diffusion in a modified polyaniline, J. Appl. Polym. Sci.: 66, 2095-2101 (1997).

Hull, D.R.; Synthetic Filament Having Antistatic Properties, US 3.803.453, Clase U.S. 361/220, 9 de abril (1974).

Kärnä, T., J. Laakso, K. Levon y E. Savolainen; Conducting Polymer Material and Method for its Production, US 5.346.649, Clase U.S. 252/500, 13 de septiembre (1994a). 
Kärnä, T. y otros ocho autores; Electrically Conductive Compositions and Methods for their Preparation, US 5.340.499, Clase U.S. 252/500, 23 de agosto (1994b).

Luzny, W., T. Kaniowski y A. Pron; Structural and transport properties of thermally processable conducting polymer: Polyaniline protonated with diphenyl phosphate, Polymer: 39 (2), 475-483 (1998).

Omastová, M., I. Chodák y J. Pionteck; Electrical and mechanical properties of conducting polymer composites, Synth. Met.: 102, 1251-1252 (1999).

Peng, Y.-Q. y otros seis autores; Einstein relation in chemically doped organic semiconductors, Appl. Phys. A: 86, 225-229 (2007).

Perento, J., Panipol-conductive polymers (2004), http://www.panipol.com, Panipol Oy, P.O. Box 163, FIN-06101 Porvoo, Finland, Panipol Oy @ 2004. Acceso: 30 de Mayo (2007).

Pomfret, S.J., P.N. Adams, N.P. Comfort y A.P. Monkman:;Electrical and mechanical properties of polyaniline fibres produced by a one-step wet spinning process, Polymer: 41, 2265-2269 (2000).

Rasnick, L.E. Jr., A.L. Belcher Jr.; Textile Effect Yarn and Method for Producing Same, US 6.332.253 B1, Clase U.S. 28/258, 25 de diciembre (2001).

Rodini, D.J.; Staple Fibers and Process for Making Them, US 5.026.603, Clase U.S. 428/362, 25 de junio (1991).

Shirakawa, H. y otros cuatro autores; Synthesis of electrically conducting polymers: Halogen derivatives of polyacetylene, $(\mathrm{CH})_{x}$, J. Chem. Soc. Chem. Comm.: (16), 578-580 (1977).

Tjong, S.C. y W. Jiang; Performance characteristics of compatibilized ternary nylon 6/ABS/LCP in-situ composites, J. Mater. Sci.: 39 (8), 2737-2746 (2004).

Tsebrenko, M.V., A.V. Yudin, T.I. Ablazova y G.V. Vinogradof; Mechanism of fibrillation in the flow of molten polymer mixtures, Polymer: 17 (9), 831-834 (1976).

Vikki, T. y otros ocho autores; Molecular recognition solvents for electrically conductive polyaniline, Macromolecules: 29, 2945-2953 (1996).

Virtanen, E. y otros siete autores; Electrically conductive compositions based on processible polyanilines-PANIPOL ${ }^{T M}$, Synth. Met.: 84, 113-114 (1997). 
